\title{
Economics of Aromatic Rice in Bastar District of Chhattisgarh, India
}

\author{
Pramita Baghel $^{1 *}$, A. K. Gauraha ${ }^{2}$ and M. R. Chandrakar ${ }^{1}$ \\ ${ }^{1}$ Department of Agricultural Economics, ${ }^{2}$ Department of Agri Business and Rural \\ Management, Indira Gandhi Krishi Vishwavidyalaya, Raipur - 492012, Chhattisgarh, India \\ *Corresponding author
}

\section{A B S T R A C T}

\section{Keywords}

Major crop of aromatic rice Badsahbhog, Cost and returns, Inputoutput ratio

\section{Article Info}

Accepted: 04 November 2019 Available Online: 10 December 2019
The present study was conducted in Bastar district of Chhattisgarh. Out of seven blocks of the district, three blocks, namely Bakawand, Tokapal and Jagdalpur were selected randomly for the study. A sample of one hundred and fifty farmers was selected for the study. The primary data from the farmers has been collected through personal interview method with the help of well designed questionnaire for the year 2014-15. The tubewell was observed as a major source of irrigation. The average cropping intensity was observed 190.60 percent. The average size of holding of aromatic rice growers was 0.43 hectares. On an average the cost of cultivation per hectare of Badsahbhog variety of aromatic rice was calculated Rs. 35368.81. Among different cost items contribution of total labour cost was found to be 43.40 percent in case of Badsahbhog variety of aromatic rice, which indicate that the contribution of labour is very high in cost of cultivation of this variety. Gross income for Basdsahbhog it was Rs.52006.02. On an average input-output ratio of aromatic rice verities of Badsahbhog came to 1:1.85 respectively. Study suggested that Cultivation of aromatic rice could be encourage by government, by providing better minimum support price

\section{Introduction}

At present, aromatic rice is recognized as high valued than normal rice in the world market. While the supply of aromatic rice does not meet its demand, consumers cherish it from paying the premium. India continue to be leading developers of new evolved aromatic rice, and the best quality of traditional Basmati and Jasmine rice cultivars are attributed by their geographical origins. Rice is an important crop grown in nearly 4.4 million ha. of land in the country with the productivity of $2.2 \mathrm{t}$./ha which is less than the productivity of many countries. In Chhattisgarh, rice occupies average of 3.75 thousand hectare with the productivity of the state ranging between 20.50 quintal per hectare depending upon the 
rainfall. The state is comprised with three agro-ecological zones i.e. Chhattisgarh plains. Bastar plateau and Northern hill region. These zones have huge variations in terms of soil topography, rainfall intensity and distribution of irrigation and adoption of agricultural production system and thus varies in the productivity of rice in these regions. Rice is grown predominantly during kharif season as rain fed crop having 2.38 .9 million hectare area but the productivity of this crop is low $8.53 \mathrm{qt} / \mathrm{ha}$ in Bastar region of Chhattisgarh. Looking to the importance of aromatic rice this study has been undertaken with specific objective. The main objective is to work out the cost and returns of aromatic rice in the study area.

\section{Materials and Methods}

Out of 27 district of Chhattisgarh state Bastar district has been selected purposely for present study.One hundred fifty farmers were selected randomly from three blocks and 50 farmers were selected from each block. The primary data were collected for the year 2014-15, which were related to cost and return of major aromatic rice The primary data was collected by the respondents using well designed schedule. To work out the cost of cultivation, returns and profit of aromatic rice standard methods of cost cultivation has been adopted to analyze the data.

\section{Results and Discussion}

General characteristic of sample
households

The general characteristic of sample households is presented in table 1 . It can be seen from the table that the average family size was 5.81. Average family size in large farm households was considerably (5.05) medium farm households (5.68), small farm households (5.87), marginal farm households was considerably (6.21) and. The literacy rate in the selected households was 75.64 per cent. Average size of holding was 1.81 hectare. It was from 0.88 hectare on marginal farm to 4.39 hectare on large farm. The sample households comprised pre - dominantly for scheduled tribe 50 per cent and scheduled caste 12 per cent. Number of working member of male was 58.77 per cent and female was 41.22 per cent.

\section{Economics of aromatic rice}

The break up cost of input factor involved in aromatic rice on the sample farmers. It reveals that the per hectare variable cost of cultivation of aromatic rice was 29025.65. The human labour share on total cost of cultivation was 43.40 per cent followed by machine power share on aromatic rice was 23.98 per cent. It was observed from table 2 that the total fixed cost and total cost were Rs 6343.16 and Rs 35368.81 respectively. The cost of cultivation was increased with the size of farm increased in aromatic rice.

\section{Cost and return on the basis of cost concept of aromatic rice}

The cost and return on the basis of cost concept in the production of aromatic rice have been presented in table 3 and 4 portrays that aromatic rice on an average cost $\mathrm{A} 1$, cost $\mathrm{A} 2$, cost B1, cost B2, cost $\mathrm{C} 1$, cost $\mathrm{C} 2$, cost C3 were worked to Rs 26862.94, Rs 26862.94, Rs 27196.11, Rs 33196.11, Rs 29368.81, Rs 35368.81 and Rs 38905.60 per hectare respectively. 
Table.1 General characteristics of sample households

\begin{tabular}{|c|c|c|c|c|c|c|}
\hline$\overline{\text { S. No }}$ & Particulars & Marginal & Small & Medium & Large & Overall \\
\hline 1. $\mathrm{I}$ & No. of total households & 38 & 54 & 41 & 17 & 150 \\
\hline 2. $\quad t$ & Average size of holding (ha) & 0.88 & 1.48 & 2.06 & 4.39 & 1.81 \\
\hline 3. $t$ & Average family size & 6.21 & 5.87 & 5.68 & 5.05 & 5.81 \\
\hline \multirow{2}{*}{$\begin{array}{ll}4 . \quad \mathrm{I} \\
\mathrm{T}\end{array}$} & Literacy & 67.38 & 77.22 & 72.85 & 100.0 & 75.64 \\
\hline & Total & $\begin{array}{c}236 \\
(100.00)\end{array}$ & $\begin{array}{c}316 \\
(100.00)\end{array}$ & $\begin{array}{c}232 \\
(100.00)\end{array}$ & $\begin{array}{c}86 \\
(100.00)\end{array}$ & $\begin{array}{c}870 \\
(100.00)\end{array}$ \\
\hline 6. $\quad$ ( & Caste wise no. of households & & & & & \\
\hline a. c & Other backward caste & $\begin{array}{c}11 \\
(28.96)\end{array}$ & $\begin{array}{c}24 \\
(44.44)\end{array}$ & $\begin{array}{c}13 \\
(31.70)\end{array}$ & $\begin{array}{l}9 \\
(52.95)\end{array}$ & $\begin{array}{c}57 \\
(38.00)\end{array}$ \\
\hline b. $\mathrm{S}$ & Scheduled tribe & $\begin{array}{c}25 \\
(65.78)\end{array}$ & $\begin{array}{c}24 \\
(44.44)\end{array}$ & $\begin{array}{c}20 \\
(48.78)\end{array}$ & $\begin{array}{c}6 \\
(35.29)\end{array}$ & $\begin{array}{c}75 \\
(50.00)\end{array}$ \\
\hline \multirow[t]{2}{*}{ c. } & Scheduled caste & $\begin{array}{c}2 \\
(5.26)\end{array}$ & $\begin{array}{c}6 \\
(11.12)\end{array}$ & $\begin{array}{c}8 \\
(19.52)\end{array}$ & $\begin{array}{c}2 \\
(11.76)\end{array}$ & $\begin{array}{c}18 \\
(12.00)\end{array}$ \\
\hline & Total & $\begin{array}{c}38 \\
(100.00)\end{array}$ & $\begin{array}{c}54 \\
(100.00)\end{array}$ & $\begin{array}{c}41 \\
(100.00)\end{array}$ & $\begin{array}{c}17 \\
(100.00)\end{array}$ & $\begin{array}{c}150 \\
(100.00)\end{array}$ \\
\hline 7. 1 & No. of working members & & & & & \\
\hline a. & Male & $\begin{array}{c}110 \\
(55.00)\end{array}$ & $\begin{array}{c}77 \\
(56.21)\end{array}$ & $\begin{array}{c}54 \\
(62.07)\end{array}$ & $\begin{array}{c}27 \\
(84.37)\end{array}$ & $\begin{array}{c}268 \\
(58.77)\end{array}$ \\
\hline \multirow[t]{2}{*}{ b. } & Female & $\begin{array}{c}90 \\
(45.00)\end{array}$ & $\begin{array}{c}60 \\
(43.79)\end{array}$ & $\begin{array}{c}33 \\
(37.93)\end{array}$ & $\begin{array}{c}5 \\
(15.63)\end{array}$ & $\begin{array}{c}188 \\
(41.23)\end{array}$ \\
\hline & Total & $\begin{array}{c}200 \\
(100.00)\end{array}$ & $\begin{array}{c}137 \\
(100.00)\end{array}$ & $\begin{array}{c}87 \\
(100.00)\end{array}$ & $\begin{array}{c}32 \\
(100.00)\end{array}$ & $\begin{array}{r}456 \\
(100.00)\end{array}$ \\
\hline
\end{tabular}

Note: figures in parentheses indicate percentage to total 
Table.2 Economics of aromatic rice on different size groups of farmers

(Rs per ha.)

\begin{tabular}{lccccc}
\hline S.No Particulars & Marginal & Small & Medium & Large & Overall \\
\hline 1. Family human labour & 3264.03 & 1810.25 & 1863.06 & 1631.37 & 2172.70 \\
& $(11.93)$ & $(6.26)$ & $(6.19)$ & $(5.31)$ & $(7.48)$ \\
2. Hired human labour & 8256.2 & 10522.02 & 11617.81 & 12135.37 & 10430.37 \\
& $(30.19)$ & $(36.42)$ & $(38.62)$ & $(39.54)$ & $(35.91)$ \\
3. Total human labour & 11520.23 & 12332.27 & 13480.87 & 13766.74 & 12603.07 \\
& $(42.12)$ & $(42.69)$ & $(44.81)$ & $(44.86)$ & $(43.40)$ \\
4. Bullock labour & 898.43 & 0 & 0 & 0 & 225.60 \\
& $(3.28)$ & $(0.00)$ & $(0.00)$ & $(0.00)$ & $(0.77)$ \\
5. Machine power & 6073.43 & 7359.92 & 7176.78 & 7204.16 & 6963.29 \\
& $(22.20)$ & $(25.48)$ & $(23.85)$ & $(23.47)$ & $(23.98)$ \\
6. Seed cost & 1408.75 & 1415.51 & 1439.88 & 1442.29 & 1434.45 \\
& $(5.15)$ & $(4.90)$ & $(4.78)$ & $(4.70)$ & $(4.94)$ \\
7. Manure \& Fertilizer & 5823.43 & 6005.14 & 6140.48 & 6320.83 & 6029.77 \\
& $(21.29)$ & $(20.78)$ & $(20.41)$ & $(20.59)$ & $(20.78)$ \\
8. Plant protection & 156.25 & 182.35 & 184.65 & 250.01 & 182.03 \\
& $(0.57)$ & $(0.63)$ & $(0.61)$ & $(0.81)$ & $(0.63)$ \\
9. Irrigation charges & 453.12 & 477.94 & 500 & 520.83 & 480.54 \\
& $(1.65)$ & $(1.65)$ & $(1.66)$ & $(1.69)$ & $(1.66)$ \\
10. Interest on working & 1013.34 & 1111.72 & 1156.90 & 1180.19 & 1106.90 \\
capital & $(3.84)$ & $(3.84)$ & $(3.84)$ & $(3.84)$ & $(3.84)$ \\
& & & & & \\
\hline
\end{tabular}

Note: figures in parentheses indicate percentage to total input cost. 
Table.3 Fixed cost of cultivation of Aromatic rice

\begin{tabular}{lccccc}
\hline & Marginal & Small & Medium & Large & Overall \\
\hline Land revenue & 10 & 10 & 10 & 10 & 10 \\
& $(0.02)$ & $(0.02)$ & $(0.02)$ & $(0.02)$ & $(0.02)$ \\
Rental value of land & 6000 & 6000 & 6000 & 6000 & 6000 \\
& $(17.82)$ & $(17.03)$ & $(16.46)$ & $(16.18)$ & $(16.96)$ \\
Interest on fixed capital & 300.5 & 320.5 & 360.5 & 380.5 & 333.16 \\
& $(0.89)$ & $(0.91)$ & $(0.98)$ & $(1.02)$ & $(0.94)$ \\
Total fixed cost (B) & 6310.5 & 6330.5 & 6370.5 & 6390.5 & 6343.16 \\
& $(18.74)$ & $(17.97)$ & $(17.47)$ & $(17.23)$ & $(17.93)$ \\
Total cost( A+B) & 33657.4 & 35215.36 & 36449.46 & 37075.55 & 35368.81 \\
& $(100.00)$ & $(100.00)$ & $(100.00)$ & $(100.00)$ & $(100.00)$ \\
\hline
\end{tabular}

Table.4 Break-up of total cost, cost concept wise

(in Rs/ ha.)

\begin{tabular}{lccccc}
\hline particular & Marginal & Small & Medium & Large & Overall \\
\hline 1. Cost A1 & 24092.87 & 27084.61 & 28225.9 & 29063.68 & 26862.94 \\
2. Cost A2 & 24092.87 & 27084.61 & 28225.9 & 29063.68 & 26862.94 \\
3. Cost B1 & 24393.37 & 27405.11 & 28586.4 & 29444.18 & 27196.11 \\
4. Cost B2 & 30393.37 & 33405.11 & 34586.4 & 35444.18 & 33196.11 \\
5. Cost C1 & 27657.4 & 29215.36 & 30449.46 & 31075.55 & 29368.81 \\
6. Cost C2 & 33657.4 & 35215.36 & 36449.46 & 37075.55 & 35368.81 \\
7. Cost C3 & 37022.78 & 38736.89 & 40094.40 & 40783.10 & 38905.60 \\
\hline
\end{tabular}

Table.5 Income over different

(in Rs/ ha.)

\begin{tabular}{lccccc}
\hline Particular & Marginal & Small & Medium & Large & Overall \\
\hline a. Cost A1 & 25957.13 & 25335.39 & 24427.1 & 24439.32 & 25143.08 \\
b. Cost A2 & 25957.13 & 25335.39 & 24427.1 & 24439.32 & 25143.08 \\
c. Cost B1 & 25656.63 & 25014.89 & 24066.6 & 24058.82 & 24809.91 \\
d. Cost B2 & 19656.63 & 19014.89 & 18066.6 & 18058.82 & 18809.91 \\
e. Cost C1 & 22392.6 & 23204.64 & 22203.54 & 22427.45 & 22637.21 \\
f. Cost C2 & 16392.6 & 17204.64 & 16203.54 & 16427.45 & 16637.21 \\
g. Cost C3 & 13027.22 & 13683.11 & 12558.6 & 12719.9 & 13100.42 \\
\hline
\end{tabular}


Table.6 Cost and return, production per quintal of the sample farms

(Rs per ha.)

\begin{tabular}{|c|c|c|c|c|c|}
\hline S.No. particulars & Marginal & Small & Medium & Large & Overall \\
\hline Input cost & 33657.4 & 35215.36 & 36449.46 & 37075.55 & 35368.81 \\
\hline 2. Gross income & 50050 & 52420 & 52653 & 53503 & 52006.02 \\
\hline Yield of main product $(\mathrm{Qt} / \mathrm{hac}$. & c.) 20.75 & 21.75 & 21.82 & 22.18 & 21.56 \\
\hline Yield of by product (Qt/hac.) & 23.25 & 24.64 & 24.72 & 24.82 & 24.33 \\
\hline 3. Net income & 16392.6 & 17204.64 & 16203.54 & 16427.45 & 16637.20 \\
\hline 4. Family labour income & 19656.63 & 19014.89 & 16203.54 & 16427.45 & 18115.78 \\
\hline 5. Farm business income & 25957.13 & 25335.39 & 24427.1 & 24439.32 & 25143.08 \\
\hline 6. Farm investment income & 22693.1 & 23525.14 & 22564.04 & 22807.95 & 22970.37 \\
\hline 7. Input - output ratio & 1: 1.83 & 1: 1.85 & $1: 1.87$ & 1: 1.90 & 1: 1.85 \\
\hline
\end{tabular}

In table 4 the average aromatic rice of income per hectare over cost $\mathrm{A} 1$, cost $\mathrm{A} 2$, cost $\mathrm{B} 1$, cost $\mathrm{B} 2$, cost $\mathrm{C} 1$, cost $\mathrm{C} 2$, cost $\mathrm{C} 3$ were calculated to Rs 25143.08, Rs 25143.08, Rs 24809.91, Rs 18809.91, Rs 22637.21, Rs 16637.21 and Rs 13100.42.

Yield, value of output, cost and measures of farm profit of aromatic rice

The value of total cost, gross income, net income, family labour income and farm business income per hectare on the sample farmers have been worked out in table 5 . clearly indicates that on an average the value input cost, gross income, net income, family labour income and farm business income are to Rs 35368.81, Rs 52006.02, Rs 16637.20, Rs 18115.78, Rs 25143.08 and Rs 22970.37 per hectare respectively (Table 6).

The input - output ratio of aromatic rice was worked out to 1: 1.85 . It is concluded that the aromatic rice variety badsahbhog was found economical in the study area. Study suggested that Cultivation of aromatic rice could be encourage by government, by providing better minimum support price.

\section{References}

Marothia, D.K., (2003) Domestic trade in aromatic rice: Lessons from an exploratory study of Chhattisgarh In: A Treatise on the Scented Rice of India. Eds: Singh R. and Singh U.S. Ludhiana \& New Delhi: Kalyani Publishers. pp. 189-210.

Shammim, Mohhamad., Singh K.K., Gangwar B., Kumar Sunil and Mandal V.P., (2013). Area and Production of Aromatic Rice Crop, Project Directorate for Farming System Research, Modhipuram - 25011.

Singh, R.K., Singh U.S. and Singh G.S., (2000). Aromatic rice's. New Delhi: Oxford \& IBH Pub. Wikipedia, the free encyclopedia.

Yadav, S.R., Chandrakar M.R., (2010). A Socio- economic Characterization of Upland Rice Production System in Dantewada District of Chhattisgarh, M.Sc (Ag.). Thesis submitted to Department of Agricultural Economics, Indira Gandhi Krishi Vishwavidyalaya, Raipur (C.G). 


\section{How to cite this article:}

Pramita Baghel, A. K. Gauraha and Chandrakar, M. R. 2019. Economics of Aromatic Rice in Bastar District of Chhattisgarh, India. Int.J.Curr.Microbiol.App.Sci. 8(12): 7-13. doi: https://doi.org/10.20546/ijcmas.2019.812.002 